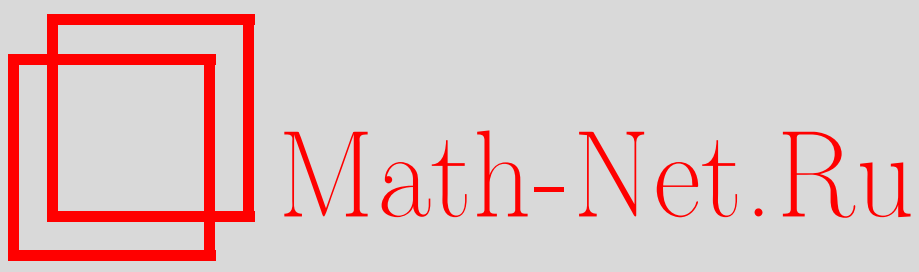

Л. Б. Миронова, Применение метода Римана к одной системе в трехмерном пространстве, Изв. вузов. Матем., 2019, номер 6, 48-57

DOI: https://doi.org/10.26907/0021-3446-2019-6-48-57

Использование Общероссийского математического портала Math-Net.Ru подразумевает, что вы прочитали и согласны с пользовательским соглашением

http://www . mathnet.ru/rus/agreement

Параметры загрузки:

IP: 3.85 .183 .62

26 апреля 2023 г., $11: 57: 26$

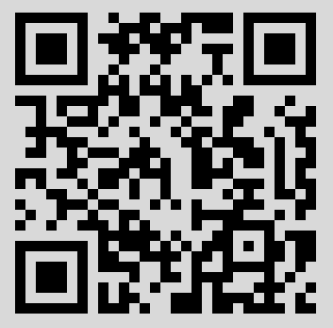


Известия вузов. Математика

2019, № 6, c. $48-57$ https://kpfu.ru/science/nauchnye-izdaniya/ivrm

e-mail: izvuz.matem@kpfu.ru

\title{
Л.Б. МИРОНОВА
}

\section{ПРИМЕНЕНИЕ МЕТОДА РИМАНА К ОДНОЙ СИСТЕМЕ В ТРЕХМЕРНОМ ПРОСТРАНСТВЕ}

\begin{abstract}
Аннотация. Для системы трех уравнений второго порядка доказаны существование и единственность решений задачи Коши и задачи с условиями на характеристиках и свободной поверхности, построены решения указанных задач в терминах матрицы Римана.
\end{abstract}

Ключевые слова: гиперболическая система, метод Римана, матрица Римана, задача Коши, характеристики.

УДК: 517.956

DOI: $10.26907 / 0021-3446-2019-6-48-57$

Введение. Система уравнений первого порядка

$$
\frac{\partial u_{i}}{\partial x_{i}}=\sum_{k=1}^{n} a_{i k}\left(x_{1}, \ldots, x_{n}\right) u_{k}+f_{i}\left(x_{1}, \ldots, x_{n}\right), \quad i=1, \ldots, n,
$$

исследовалась многими авторами (например, [1]-[3] и литература при этих статьях). Система (1) представляет интерес, в частности, с точки зрения применения получаемых результатов к изучению важных в теоретическом и практическом отношении дифференциальных уравнений смешанного типа. Наибольшее число публикаций относится к случаю, когда в (1) $n=2$.

В работе [4] предложен вариант метода Римана для системы дифференциальных уравнений с кратными характеристиками, в терминах матрицы Римана построены решения задач Коши и Гурса. В статье [5] метод Римана применяется для исследования задач для одной системы уравнений с двумя независимыми переменными с кратными характеристиками. Системы гиперболических дифференциальных уравнений в последнее время исследовались рядом авторов в различных направлениях [6]-[10].

В ряде работ В.И. Жегалова и его учеников был разработан метод Римана для класса уравнений с доминирующими частными производными

$$
\left(D_{1}+D_{2}\right) u=f\left(x_{1}, \ldots, x_{n}\right),
$$

где

$$
D_{1} \equiv \frac{\partial^{k_{1}+\cdots+k_{n}}}{\partial x_{1}^{k_{1}} \ldots \partial x_{n}^{k_{n}}},
$$

а $D_{2}$ - линейный дифференциальный оператор с переменными коэффициентами, содержащий лишь производные, получаемые из $D_{1}$ отбрасыванием по крайней мере одного дифференцирования [11]-[16]. Уравнение (2) имеет приложения в теориях фильтрации жидкости в

Поступила в редакцию 24.04.2018, после доработки 24.04.2018. Принята к публикации 26.09.2018 
трещиноватых средах, поглощения влаги корнями растений, колебаний стержней с учетом эффектов поперечной инерции, распространения волн в диспергирующих средах.

Отметим также работы Р.К.Романовского, Е.В.Воробьевой, М.В. Мендзив, Ю.А. Медведева, в которых исследованы начально-краевые задачи и задачи оптимального управления для гиперболических систем (в том числе с использованием определенных В.К. Романовским матриц Римана), устойчивость и экспоненциальная дихотомия решений [17]-[21].

В данной статье метод Римана применяется для исследования задачи Коши и задачи с условиями на характеристической и свободной части границы (смешанная задача) для системы уравнений с тремя независимыми переменными

$$
\begin{aligned}
& u_{x x}=a_{1}(x, y, z) v_{x}+b_{1}(x, y, z) w_{x}+c_{1}(x, y, z) u+d_{1}(x, y, z) v+e_{1}(x, y, z) w+f_{1}(x, y, z), \\
& v_{y y}=a_{2}(x, y, z) u_{y}+b_{2}(x, y, z) w_{y}+c_{2}(x, y, z) u+d_{2}(x, y, z) v+e_{2}(x, y, z) w+f_{2}(x, y, z), \\
& w_{z z}=a_{3}(x, y, z) u_{z}+b_{3}(x, y, z) v_{z}+c_{3}(x, y, z) u+d_{3}(x, y, z) v+e_{3}(x, y, z) w+f_{3}(x, y, z) .
\end{aligned}
$$

Считаем, что в замыкании рассматриваемой области $D$ пространства $(x, y, z)$ выполняются включения $a_{i}, b_{i} \in C^{2}, c_{i}, d_{i}, e_{i}, f_{i} \in C^{1}, i=\overline{1, n}$. Решение (3) класса $u, v, w \in C^{1}(D), u_{x x}$, $v_{y y}, w_{z z} \in C(D)$ назовем регулярным в $D$.

К системе (3) подстановками

$$
\begin{gathered}
u^{*}=\exp \left(\frac{1}{2} \int_{x_{0}}^{x} a_{1}^{*}(\alpha, y, z) d \alpha\right) u, \quad v^{*}=\exp \left(\frac{1}{2} \int_{y_{0}}^{y} b_{2}^{*}(x, \beta, z) d \beta\right) v \\
w^{*}=\exp \left(\frac{1}{2} \int_{z_{0}}^{z} c_{3}^{*}(x, y, \gamma) d \gamma\right) w
\end{gathered}
$$

сводится система со старшими производными вида

$$
\begin{gathered}
u_{x x}^{*}=a_{1}^{*}(x, y, z) u_{x}^{*}+b_{1}^{*}(x, y, z) v_{x}^{*}+c_{1}^{*}(x, y, z) w_{x}^{*}+d_{1}^{*}(x, y, z) u^{*}+ \\
+e_{1}^{*}(x, y, z) v^{*}+f_{1}^{*}(x, y, z) w^{*}+g_{1}^{*}(x, y, z), \\
v_{y y}^{*}=a_{2}^{*}(x, y, z) u_{y}^{*}+b_{2}^{*}(x, y, z) v_{y}^{*}+c_{2}^{*}(x, y, z) w_{y}^{*}+d_{2}^{*}(x, y, z) u^{*}+ \\
+e_{2}^{*}(x, y, z) v^{*}+f_{2}^{*}(x, y, z) w^{*}+g_{2}^{*}(x, y, z), \\
w_{z z}^{*}=a_{3}^{*}(x, y, z) u_{z}^{*}+b_{3}^{*}(x, y, z) v_{z}^{*}+c_{3}^{*}(x, y, z) w_{z}^{*}+d_{3}^{*}(x, y, z) u^{*}+ \\
+e_{3}^{*}(x, y, z) v^{*}+f_{3}^{*}(x, y, z) w^{*}+g_{3}^{*}(x, y, z) .
\end{gathered}
$$

1. Задача Коши. Пусть $S: z=\sigma(x, y)$ - поверхность класса $C^{2}$ в пространстве $(x, y, z)$, причем $S$ в каждой своей точке имеет касательную плоскость, не параллельную ни одной из координатных осей. Пусть для определенности $\sigma_{x}^{\prime}<0, \sigma_{y}^{\prime}<0$. Проведем через точку $P\left(x_{0}, y_{0}, z_{0}\right)$ плоскости $x=x_{0}, y=y_{0}, z=z_{0}$, которые пересекают поверхность $S$ по кривым $B C, C A$ и $A B$ соответственно. Плоскости $x=x_{0}, y=y_{0}, z=z_{0}$ и поверхность $S$ определяют область $D$, граница которой состоит из двумерных многообразий $A P C, B C P, B P A$ и $A C B$. Ориентацию $D$ считаем положительной.

Задача Коши: найти регулярное в D решение системы (3), удовлетворлющее на поверхности $S$ условиям

$$
\begin{aligned}
\left.u\right|_{S}=u_{0}(x, y), & \left.\frac{\partial u}{\partial n}\right|_{S}=u_{10}(x, y),\left.\quad v\right|_{S}=v_{0}(x, y), \\
\left.\frac{\partial v}{\partial n}\right|_{S}=v_{10}(x, y), & \left.w\right|_{S}=w_{0}(x, y),\left.\quad \frac{\partial w}{\partial n}\right|_{S}=w_{10}(x, y) .
\end{aligned}
$$

Здесь $\vec{n}=\left(n_{1}, n_{2}, n_{3}\right)$ - единичный вектор внешней нормали к поверхности $S, u_{0}, v_{0}, w_{0} \in$ $C^{2}, u_{10}, v_{10}, w_{10} \in C^{1}$. 
Отметим, что поверхность $S$ можно задать уравнениями $y=\sigma^{*}(x, z), x=\sigma^{* *}(y, z)$. Очевидно, условия (4) могут быть записаны в следующих формах:

$$
\begin{aligned}
& \left.u\right|_{S}=u_{0}^{*}(x, z),\left.\quad \frac{\partial u}{\partial n}\right|_{S}=u_{10}^{*}(x, z),\left.\quad v\right|_{S}=v_{0}^{*}(x, z), \\
& \left.\frac{\partial v}{\partial n}\right|_{S}=v_{10}^{*}(x, z),\left.\quad w\right|_{S}=w_{0}^{*}(x, z),\left.\quad \frac{\partial w}{\partial n}\right|_{S}=w_{10}^{*}(x, z) ; \\
& \left.u\right|_{S}=u_{0}^{* *}(y, z),\left.\quad \frac{\partial u}{\partial n}\right|_{S}=u_{10}^{* *}(y, z),\left.\quad v\right|_{S}=v_{0}^{* *}(y, z), \\
& \left.\frac{\partial v}{\partial n}\right|_{S}=v_{10}^{* *}(y, z),\left.\quad w\right|_{S}=w_{0}^{* *}(y, z),\left.\quad \frac{\partial w}{\partial n}\right|_{S}=w_{10}^{* *}(y, z) .
\end{aligned}
$$

Обозначим $w_{1}(x, y, \sigma(x, y))=\bar{w}_{1}(x, y), v_{1}\left(x, \sigma^{*}(x, z), z\right)=\bar{v}_{1}^{*}(x, z), u_{1}\left(\sigma^{* *}(y, z), y, z\right)=$ $\bar{u}_{1}^{* *}(y, z)$.

Условия (4) (или эквивалентные им (5), (6)) позволяют определить значения частных производных первого порядка функций $u, v, w$ на $S$. Действительно, например, для $w$ имеем на поверхности $S$ равенства

$$
w_{x}+w_{z} \sigma_{x}=w_{0 x}, \quad w_{y}+w_{z} \sigma_{y}=w_{0 y}, \quad w_{x} n_{1}+w_{y} n_{2}+w_{z} n_{3}=w_{10} .
$$

Определитель системы (7)

$$
\left|\begin{array}{ccc}
1 & 0 & \sigma_{x} \\
0 & 1 & \sigma_{y} \\
n_{1} & n_{2} & n_{3}
\end{array}\right| \neq 0
$$

так как первые две строки определителя образуют координаты векторов, касательных к $S$, а третья образована координатами $\vec{n}$. Поэтому величина $\bar{w}_{1}(x, y)$ известна в силу (4). Аналогично, будут известными величины $\bar{v}_{1}^{*}(x, z), \bar{u}_{1}^{* *}(y, z)$.

Докажем существование и единственность решения задачи Коши (3), (4). Преобразуем (3) к виду

$$
\begin{array}{ll}
u_{x}=u_{1}+a_{1} v+b_{1} w, & u_{1 x}=c_{1} u+\left(d_{1}-a_{1 x}\right) v+\left(e_{1}-b_{1 x}\right) w+f_{1}, \\
v_{y}=a_{2} u+v_{1}+b_{2} w, & v_{1 y}=\left(c_{2}-a_{2 y}\right) u+d_{2} v+\left(e_{2}-b_{2 y}\right) w+f_{2}, \\
w_{z}=a_{3} u+b_{3} v+w_{1}, & w_{1 z}=\left(c_{3}-a_{3 z}\right) u+\left(d_{3}-b_{3 z}\right) v+e_{3} w+f_{3} .
\end{array}
$$

Обозначим $d_{10}=d_{1}-a_{1 x}, e_{10}=e_{1}-b_{1 x}, c_{20}=c_{2}-a_{2 y}, e_{20}=e_{2}-b_{2 y}, c_{30}=c_{3}-a_{3 z}, d_{30}=$ $d_{3}-b_{3 z}$. Сведем систему (8) с учетом условий (4)-(6) к системе интегральных уравнений

$$
\begin{gathered}
u(x, y, z)=u_{0}^{* *}(y, z)+\int_{\sigma^{* *}(y, z)}^{x}\left(u_{1}+a_{1} v+b_{1} w\right)(\alpha, y, z) d \alpha, \\
u_{1}(x, y, z)=\bar{u}_{1}^{* *}(y, z)+\int_{\sigma^{* *}(y, z)}^{x}\left(c_{1} u+d_{10} v+e_{10} w+f_{1}\right)(\alpha, y, z) d \alpha, \\
v(x, y, z)=v_{0}^{*}(x, z)+\int_{\sigma^{*}(x, z)}^{y}\left(v_{1}+a_{2} u+b_{2} w\right)(x, \beta, z) d \beta,
\end{gathered}
$$




$$
\begin{gathered}
v_{1}(x, y, z)=\bar{v}_{1}^{*}(x, z)+\int_{\sigma^{*}(x, z)}^{y}\left(c_{20} u+d_{2} v+e_{20} w+f_{2}\right)(x, \beta, z) d \beta, \\
w(x, y, z)=w_{0}(x, y)+\int_{\sigma(x, y)}^{z}\left(w_{1}+a_{3} u+b_{3} v\right)(x, y, \gamma) d \gamma, \\
w_{1}(x, y, z)=\bar{w}_{1}(x, y)+\int_{\sigma(x, y)}^{z}\left(c_{30} u+d_{30} v+e_{3} w+f_{3}\right)(x, y, \gamma) d \gamma .
\end{gathered}
$$

Решение системы интегральных уравнений Вольтерра (9) существует и единственно в классе непрерывных функций.

Докажем эквивалентность задачи Коши (3), (4) и системы (9). Очевидно, система (9) - следствие (3), (4). Обратно, дифференцируя первое уравнение системы (9), получаем $u_{x}=u_{1}$. Кроме того, $\left.u\right|_{S}=u_{0}^{* *}(y, z)$, что эквивалентно выполнению условия $\left.u\right|_{S}=u_{0}(x, y)$. Дифференцируем теперь второе уравнение системы (9) по $x$, в результате получаем первое уравнение (3). При этом $\left.u_{1}\right|_{S}=\bar{u}_{1}^{* *}(y, z)$. Аналогично получаем оставшиеся два уравнения системы (3) и граничные условия $\left.v\right|_{S}=v_{0}^{*}(x, z),\left.w\right|_{S}=w_{0}(x, y),\left.v_{1}\right|_{S}=\bar{v}_{1}^{*}(x, z)$, $\left.w_{1}\right|_{S}=\bar{w}_{1}(x, y)$. По известным значениям $\left.u_{1}\right|_{S},\left.v\right|_{S},\left.w\right|_{S}$ определяется $\left.u_{x}\right|_{S}$. На $S$ справедливы соотношения

$$
u_{y}+u_{x} \sigma_{y}^{* *}=u_{0 y}^{* *}, \quad u_{z}+u_{x} \sigma_{z}^{* *}=u_{0 z}^{* *}, \quad u_{x} n_{1}+u_{y} n_{2}+u_{z} n_{3}=\left.\frac{\partial u}{\partial n}\right|_{S},
$$

из которых по известным $\left.u\right|_{S},\left.u_{x}\right|_{S}$ определяются $u_{y}, u_{z}$ на $S$, а следовательно, и

$$
\left.\frac{\partial u}{\partial n}\right|_{S}=u_{10}
$$

Аналогично получаем предписанные (4) значения $\partial v /\left.\partial n\right|_{S}, \partial w /\left.\partial n\right|_{S}$. Эквивалентность задачи Коши и системы (9) доказана.

Теорема 1. Если в замыкании области $D$ выполняются включения $a_{i}, b_{i} \in C^{2}, c_{i}, d_{i}, e_{i}$, $f_{i} \in C^{1}, i=\overline{1,3}$, то решение задачи Коши (3), (4) существует и единственно.

2. Смешанная задача. Пусть область $D_{0}=\left\{x_{0}<x<x_{1}, y_{0}<y<y_{1}, z_{0}<z<z_{1}\right\}$ представляет собой характеристический параллелепипед в пространстве $(x, y, z)$ с отсеченным нехарактеристической поверхностью $S: z=\sigma(x, y)$ класса $C^{2}$ трехгранным углом с вершиной в точке $\left(x_{0}, y_{0}, z_{0}\right)$ (рис. 1$)$. Для определенности полагаем $x_{0} \geqslant 0, y_{0} \geqslant 0, z_{0} \geqslant 0$, $\sigma_{x}^{\prime}<0, \sigma_{y}^{\prime}<0$. Обозначим через $X, Y, Z$ грани $D_{0}$ при $x=x_{0}, y=y_{0}, z=z_{0}$ соответственно.

Требуется найти регулярное в $D_{0}$ решение $(3)$, удовлетворяющее условиям

$$
\begin{gathered}
\left.u\right|_{S \cup \bar{X}}=u_{0}(y, z),\left.\quad v\right|_{S \cup \bar{Y}}=v_{0}(x, z),\left.\quad w\right|_{S \cup \bar{Z}}=w_{0}(x, y), \\
\left.\frac{\partial u}{\partial n}\right|_{S}=u_{10}(y, z),\left.\quad \frac{\partial v}{\partial n}\right|_{S}=v_{10}(x, z),\left.\quad \frac{\partial w}{\partial n}\right|_{S}=w_{10}(x, y), \\
\left.\left(u_{x}-a_{1} v-b_{1} w\right)\right|_{\bar{X}}=\varphi_{1}(y, z),\left.\quad\left(v_{y}-a_{2} u-b_{2} w\right)\right|_{\bar{Y}}=\varphi_{2}(x, z), \\
\left.\left(w_{z}-a_{3} u-b_{3} v\right)\right|_{\bar{Z}}=\varphi_{3}(x, y),
\end{gathered}
$$

$\vec{n}$ - внешняя нормаль к $S, u_{0} \in C^{1}(S \cup \bar{X}), u_{10} \in C^{2}(S \cup \bar{X}), \varphi_{1} \in C^{1}(\bar{X}), v_{0} \in C^{1}(S \cup \bar{Y})$, $v_{10} \in C^{2}(S \cup \bar{Y}), \varphi_{2} \in C^{1}(\bar{Y}), w_{0} \in C^{1}(S \cup \bar{Z}), w_{10} \in C^{2}(S \cup \bar{Z}), \varphi_{3} \in C^{1}(\bar{Z})$. Кроме того, должны выполняться условия согласования $u_{x} \in C(S \cup \bar{X}), v_{y} \in C(S \cup \bar{Y}), w_{z} \in C(S \cup \bar{Z})$. 


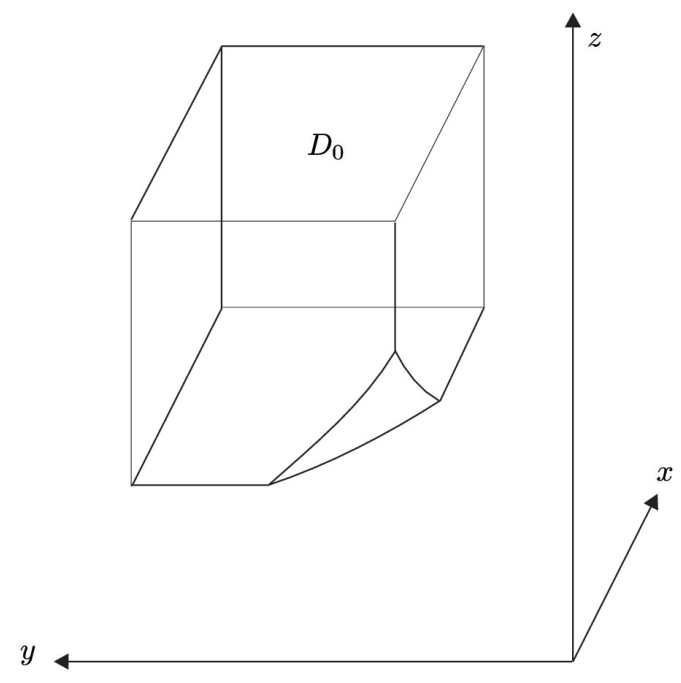

Рис. 1.

Пусть многообразия $S \cup \bar{X}, S \cup \bar{Y}, S \cup \bar{Z}$ имеют соответственно уравнения $x=\sigma_{1}(y, z)$, $y=\sigma_{2}(x, z), z=\sigma_{3}(x, y)$. Обозначим

$$
\begin{aligned}
& \chi_{1}(y, z)=\left\{\begin{array}{l}
\varphi_{1}(y, z) \text { на } \bar{X} ; \\
\left(u_{x}-a_{1} v-b_{1} w\right)\left(\sigma_{1}(y, z), y, z\right) \text { на } S,
\end{array}\right. \\
& \chi_{2}(x, z)=\left\{\begin{array}{l}
\varphi_{2}(x, z) \text { на } \bar{Y} ; \\
\left(v_{y}-a_{2} u-b_{2} w\right)\left(x, \sigma_{2}(x, z), z\right) \text { на } S,
\end{array}\right. \\
& \chi_{3}(x, y)=\left\{\begin{array}{l}
\varphi_{3}(x, y) \text { на } \bar{Z} ; \\
\left(w_{z}-a_{3} u-b_{3} v\right)\left(x, y, \sigma_{3}(x, y)\right) \text { на } S .
\end{array}\right.
\end{aligned}
$$

Докажем существование и единственность решения смешанной задачи (3), (10). Сведем систему (8) с условиями (10) к системе интегральных уравнений

$$
\begin{gathered}
u(x, y, z)=u_{0}(y, z)+\int_{\sigma_{1}(y, z)}^{x}\left(u_{1}+a_{1} v+b_{1} w\right)(\alpha, y, z) d \alpha, \\
u_{1}(x, y, z)=\chi_{1}(y, z)+\int_{\sigma_{1}(y, z)}^{x}\left(c_{1} u+d_{10} v+e_{10} w+f_{1}\right)(\alpha, y, z) d \alpha, \\
v(x, y, z)=v_{0}(x, z)+\int_{\sigma_{2}(x, z)}^{y}\left(v_{1}+a_{2} u+b_{2} w\right)(x, \beta, z) d \beta, \\
v_{1}(x, y, z)=\chi_{2}(x, z)+\int_{\sigma_{2}(x, z)}^{y}\left(c_{20} u+d_{2} v+e_{20} w+f_{2}\right)(x, \beta, z) d \beta,
\end{gathered}
$$




$$
\begin{gathered}
w(x, y, z)=w_{0}(x, y)+\int_{\sigma_{3}(x, y)}^{z}\left(w_{1}+a_{3} u+b_{3} v\right)(x, y, \gamma) d \gamma \\
w_{1}(x, y, z)=\chi_{3}(x, y)+\int_{\sigma_{3}(x, y)}^{z}\left(c_{30} u+d_{30} v+e_{3} w+f_{3}\right)(x, y, \gamma) d \gamma
\end{gathered}
$$

Решение системы (11) существует и единственно в классе непрерывных функций. Эквивалентность смешанной задачи и системы (11) доказывается так же, как и в случае задачи Коши.

Теорема 2. Если в замыкании области $D_{0}$ выполняются включения $a_{i}, b_{i} \in C^{2}, c_{i}, d_{i}, e_{i}$, $f_{i} \in C^{1}, i=\overline{1,3}$, то решение смешанной задачи (3), (10) существует и единственно.

3. Построение решений задач в терминах матрицы Римана. Перейдем к выводу формул для решений задачи Коши и смешанной задачи в терминах матрицы Римана. Перепишем (8) в векторно-матричной форме

$$
\begin{aligned}
& L(\mathbf{U})=\mathbf{F}, \quad L(\mathbf{U}) \equiv \mathbf{A}_{1} \mathbf{U}_{x}+\mathbf{A}_{2} \mathbf{U}_{y}+\mathbf{A}_{3} \mathbf{U}_{z}-\mathbf{B U}, \\
& \mathbf{U}=\operatorname{colon}\left(u, u_{1}, v, v_{1}, w, w_{1}\right), \\
& \mathbf{A}_{1}=\left(\begin{array}{cccccc}
1 & 0 & 0 & 0 & 0 & 0 \\
0 & 1 & 0 & 0 & 0 & 0 \\
0 & 0 & 0 & 0 & 0 & 0 \\
0 & 0 & 0 & 0 & 0 & 0 \\
0 & 0 & 0 & 0 & 0 & 0 \\
0 & 0 & 0 & 0 & 0 & 0
\end{array}\right), \quad \mathbf{A}_{2}=\left(\begin{array}{cccccc}
0 & 0 & 0 & 0 & 0 & 0 \\
0 & 0 & 0 & 0 & 0 & 0 \\
0 & 0 & 1 & 0 & 0 & 0 \\
0 & 0 & 0 & 1 & 0 & 0 \\
0 & 0 & 0 & 0 & 0 & 0 \\
0 & 0 & 0 & 0 & 0 & 0
\end{array}\right), \\
& \mathbf{A}_{3}=\left(\begin{array}{cccccc}
0 & 0 & 0 & 0 & 0 & 0 \\
0 & 0 & 0 & 0 & 0 & 0 \\
0 & 0 & 0 & 0 & 0 & 0 \\
0 & 0 & 0 & 0 & 0 & 0 \\
0 & 0 & 0 & 0 & 1 & 0 \\
0 & 0 & 0 & 0 & 0 & 1
\end{array}\right), \quad \mathbf{B}=\left(\begin{array}{cccccc}
0 & 1 & a_{1} & 0 & b_{1} & 0 \\
c_{1} & 0 & d_{10} & 0 & e_{10} & 0 \\
a_{2} & 0 & 0 & 1 & b_{2} & 0 \\
c_{20} & 0 & d_{2} & 0 & e_{20} & 0 \\
a_{3} & 0 & b_{3} & 0 & 0 & 1 \\
c_{30} & 0 & d_{30} & 0 & e_{3} & 0
\end{array}\right), \\
& \mathbf{F}=\operatorname{colon}\left(0, f_{1}, 0, f_{2}, 0, f_{3}\right) .
\end{aligned}
$$

Введем матрицу Римана $\mathbf{R}=\operatorname{colon}\left(\mathbf{R}_{1}, \mathbf{R}_{2}, \mathbf{R}_{3}, \mathbf{R}_{4}, \mathbf{R}_{5}, \mathbf{R}_{6}\right)$, где $\mathbf{R}_{i}(x, y, z, \xi, \eta, \zeta)=\left(r_{i 1}, r_{i 2}\right.$, $\left.r_{i 3}, r_{i 4}, r_{i 5}, r_{i 6}\right), i=\overline{1,6}$, являются решениями систем

$$
\begin{gathered}
r_{i 1}(x, y, z)=\delta_{i 1}-\int_{\xi}^{x}\left(c_{1} r_{i 2}+a_{2} r_{i 3}+c_{20} r_{i 4}+a_{3} r_{i 5}+c_{30} r_{i 6}\right)(\alpha, y, z) d \alpha, \\
r_{i 2}(x, y, z)=\delta_{i 2}-\int_{\xi}^{x} r_{i 1}(\alpha, y, z) d \alpha \\
r_{i 3}(x, y, z)=\delta_{i 3}-\int_{\eta}^{y}\left(a_{1} r_{i 1}+d_{10} r_{i 2}+d_{2} r_{i 4}+b_{3} r_{i 5}+d_{30} r_{i 6}\right)(x, \beta, z) d \beta,
\end{gathered}
$$




$$
\begin{gathered}
r_{i 4}(x, y, z)=\delta_{i 4}-\int_{\eta}^{y} r_{i 3}(x, \beta, z) d \beta \\
r_{i 5}(x, y, z)=\delta_{i 5}-\int_{\zeta}^{z}\left(b_{1} r_{i 1}+e_{10} r_{i 2}+b_{2} r_{i 3}+e_{20} r_{i 4}+e_{3} r_{i 6}\right)(x, y, \gamma) d \gamma \\
r_{i 6}(x, y, z)=\delta_{i 6}-\int_{\zeta}^{z} r_{i 5}(x, y, \gamma) d \gamma
\end{gathered}
$$

$\delta_{i j}$ - символ Кронекера. Решения систем (13) при каждом $i$ существуют и единственны в классе непрерывных функций. По первой тройке аргументов $(x, y, z)$ матрица $\mathbf{R}$ удовлетворяет сопряженной к (12) системе $L^{*}(\mathbf{V})=0, \quad L^{*}(\mathbf{V}) \equiv-\left(\mathbf{V A}_{1}\right)_{x}-\left(\mathbf{V A}_{2}\right)_{y}-(\mathbf{V A})_{z}-\mathbf{V B}$.

Справедливо тождество

$$
\mathbf{R} L(\mathbf{U})=\left(\mathbf{R} \mathbf{A}_{1} \mathbf{U}\right)_{x}+\left(\mathbf{R} \mathbf{A}_{2} \mathbf{U}\right)_{y}+\left(\mathbf{R} \mathbf{A}_{3} \mathbf{U}\right)_{z},
$$

которое может быть проверено непосредственно. Интегрированием (14) получим решения задачи Коши и смешанной задачи.

Построим решение задачи Коши. Рассмотрим точку $M(\xi, \eta, \zeta) \in D$. Пусть для определенности $\xi>0, \eta>0, \zeta>0, \sigma_{x}(x, y)<0, \sigma_{y}(x, y)<0$. Проведем через $M$ плоскости $x=\xi$, $y=\eta, z=\zeta$, пересекающие поверхность $S$ по кривым $B_{1} C_{1}, C_{1} A_{1}$ и $A_{1} B_{1}$ соответственно. Плоскости $x=\xi, y=\eta, z=\zeta$ и поверхность $S$ определяют область $D_{1} \subset D$, граница которой состоит из двумерных многообразий $A_{1} M C_{1}, B_{1} C_{1} M, B_{1} M A_{1}$ и $A_{1} C_{1} B_{1}$.

Первая строка (14) дает

$$
r_{12} f_{1}+r_{14} f_{2}+r_{16} f_{3}=\left(r_{11} u+r_{12} u_{1}\right)_{x}+\left(r_{13} v+r_{14} v_{1}\right)_{y}+\left(r_{15} w+r_{16} w_{1}\right)_{z},
$$

где $r_{1 j}=r_{1 j}(x, y, z, \xi, \eta, \zeta)$, остальные функции зависят от $(x, y, z)$. В силу (13)

$$
\begin{aligned}
r_{11}(\xi, \beta, \gamma, \xi, \eta, \zeta) \equiv 1, \quad r_{12}(\xi, \beta, \gamma, \xi, \eta, \zeta) & =r_{13}(\alpha, \eta, \gamma, \xi, \eta, \zeta)= \\
= & r_{14}(\alpha, \eta, \gamma, \xi, \eta, \zeta)=r_{15}(\alpha, \beta, \zeta, \xi, \eta, \zeta)=r_{16}(\alpha, \beta, \zeta, \xi, \eta, \zeta) \equiv 0
\end{aligned}
$$

Интегрируем (15) по области $D_{1}$

$$
\begin{array}{r}
\iint_{M B_{1} C_{1}}\left(r_{11} u+r_{12} u_{1}\right)(\xi, \beta, \gamma, \xi, \eta, \zeta) d \beta \wedge d \gamma+\iint_{C_{1} A_{1} M}\left(r_{13} v+r_{14} v_{1}\right)(\alpha, \eta, \gamma, \xi, \eta, \zeta) d \gamma \wedge d \alpha+ \\
+\iint_{M A_{1} B_{1}}\left(r_{15} w+r_{16} w_{1}\right)(\alpha, \beta, \zeta, \xi, \eta, \zeta) d \alpha \wedge d \beta+\iint_{B_{1} A_{1} C_{1}}\left(r_{11} u+r_{12} u_{1}\right)(x, \beta, \gamma, \xi, \eta, \zeta) d \beta \wedge d \gamma+ \\
+\left(r_{13} v+r_{14} v_{1}\right)(\alpha, y, \gamma, \xi, \eta, \zeta) d \gamma \wedge d \alpha+\left(r_{15} w+r_{16} w_{1}\right)(\alpha, \beta, z, \xi, \eta, \zeta) d \alpha \wedge d \beta= \\
=\iiint_{D_{1}}\left(r_{12} f_{1}+r_{14} f_{2}+r_{16} f_{3}\right)(\alpha, \beta, \gamma, \xi, \eta, \zeta) d \alpha d \beta d \gamma
\end{array}
$$

С учетом (16) получаем

$$
\iint_{M B_{1} C_{1}} u(\xi, \beta, \gamma) d \beta \wedge d \gamma=h_{1}(\xi, \eta, \zeta)
$$

$h_{1}(\xi, \eta, \zeta)$ выражается через $\mathbf{R}$ и данные Коши (4). Дифференцирование (17) в рассматриваемом случае дает

$$
u(\xi, \eta, \zeta)=\frac{\partial^{2} h_{1}(\xi, \eta, \zeta)}{\partial \eta \partial \zeta}
$$


Аналогично, используя третью и пятую строки (14), получаем выражения для $v(\xi, \eta, \zeta)$ и $w(\xi, \eta, \zeta)$ :

$$
v(\xi, \eta, \zeta)=\frac{\partial^{2} h_{2}(\xi, \eta, \zeta)}{\partial \xi \partial \zeta}, \quad w(\xi, \eta, \zeta)=\frac{\partial^{2} h_{3}(\xi, \eta, \zeta)}{\partial \xi \partial \eta} .
$$

При этом надо в $(18)$ заменить $r_{1 j}$ на $r_{3 j}$ и $r_{5 j}$ соответственно. Используя вторую, четвертую и шестую строки (14) можно определить $u_{1}, v_{1}, w_{1}$.

Заметим, что значения $\left.u\right|_{S},\left.u_{1}\right|_{S},\left.v\right|_{S},\left.v_{1}\right|_{S},\left.w\right|_{S},\left.w_{1}\right|_{S}$, требуемые для получения $u(\xi, \eta, \zeta)$, $v(\xi, \eta, \zeta), w(\xi, \eta, \zeta)$ по формулам $(18),(19)$, определяются из (4). Обратно, функции $u, v, w$, определяемые по (18), (19), и, определяемые из аналогичных формул, функции $u_{1}, v_{1}, w_{1}$ удовлетворяют на $S$ условиям

$$
\begin{gathered}
\left.u\right|_{S}=u_{0}(x, y),\left.\quad v\right|_{S}=v_{0}(x, y),\left.\quad w\right|_{S}=w_{0}(x, y), \\
\left.u_{1}\right|_{S}=u_{x}(x, y, \sigma(x, y))-a_{1}(x, y, \sigma(x, y)) v_{0}(x, y)-b_{1}(x, y, \sigma(x, y)) w_{0}(x, y), \\
\left.v_{1}\right|_{S}=v_{y}(x, y, \sigma(x, y))-a_{2}(x, y, \sigma(x, y)) u_{0}(x, y)-b_{2}(x, y, \sigma(x, y)) w_{0}(x, y), \\
\left.w_{1}\right|_{S}=w_{z}(x, y, \sigma(x, y))-a_{3}(x, y, \sigma(x, y)) u_{0}(x, y)-b_{3}(x, y, \sigma(x, y)) v_{0}(x, y) .
\end{gathered}
$$

Чтобы доказать, что найденные функции $u, v, w$ удовлетворяют (4), осталось показать, что по (20) могут быть восстановлены значения $\partial u /\left.\partial n\right|_{S}, \partial v /\left.\partial n\right|_{S}, \partial w /\left.\partial n\right|_{S}$. Знание $\left.v\right|_{S},\left.w\right|_{S}$ и $\left.u_{1}\right|_{S}$ позволяет определить из $(20) u_{x}(x, y, \sigma(x, y))$. На $S$ справедливы соотношения

$$
u_{x}+u_{z} \sigma_{x}=u_{0 x}, \quad u_{y}+u_{z} \sigma_{y}=u_{0 y}, \quad u_{x} n_{1}+u_{y} n_{2}+u_{z} n_{3}=\frac{\partial u}{\partial n} .
$$

Первые два уравнения (21) позволяют выразить $u_{y}$ и $u_{z}$ на $S$ через $u_{0 x}, u_{0 y}$ и $u_{x}(x, y, \sigma(x, y))=$ $\left.u_{x}\right|_{S}$, а третье может восстановить значение $\partial u /\left.\partial n\right|_{S}=u_{10}(x, y)$. Аналогично рассуждаем и относительно функций $v$ и $w$.

Таким образом, формулы (18), (19) действительно определяют решение задачи Коши.

Перейдем к построению решения смешанной задачи. Рассмотрим точку $M(\xi, \eta, \zeta) \in D_{0}$. Проведем через $M$ плоскости $x=\xi, y=\eta, z=\zeta$. Эти плоскости определяют область $D_{1} \subset D$, граница которой состоит из двумерных многообразий $S=A B C, A E P F B \subset$ $\bar{X}, A C G Q E \subset \bar{Y}, C B F T G \subset \bar{Z}, G T M Q \subset\{(x, y, z) \mid x=\xi\}, P M T F \subset\{(x, y, z) \mid y=\eta\}$, $E Q M P \subset\{(x, y, z) \mid z=\zeta\}$.

Интегрируем (15) по области $D_{1}$

$$
\begin{aligned}
& \iint_{G T M Q}\left(r_{11} u+r_{12} u_{1}\right)(\xi, \beta, \gamma, \xi, \eta, \zeta) d \beta \wedge d \gamma+\iint_{T F P M}\left(r_{13} v+r_{14} v_{1}\right)(\alpha, \eta, \gamma, \xi, \eta, \zeta) d \gamma \wedge d \alpha+ \\
+ & \iint_{E Q M P}\left(r_{15} w+r_{16} w_{1}\right)(\alpha, \beta, \zeta, \xi, \eta, \zeta) d \alpha \wedge d \beta+\iint_{A E P F B}\left(r_{11} u+r_{12} u_{1}\right)(x, \beta, \gamma, \xi, \eta, \zeta) d \beta \wedge d \gamma+ \\
+ & \iint_{A C G Q E}\left(r_{13} v+r_{14} v_{1}\right)(\alpha, y, \gamma, \xi, \eta, \zeta) d \gamma \wedge d \alpha+\iint_{B F T G C}\left(r_{15} w+r_{16} w_{1}\right)(\alpha, \beta, z, \xi, \eta, \zeta) d \alpha \wedge d \beta+ \\
& +\iint_{A B C}\left(r_{11} u+r_{12} u_{1}\right)(x, \beta, \gamma, \xi, \eta, \zeta) d \beta \wedge d \gamma+\left(r_{13} v+r_{14} v_{1}\right)(\alpha, y, \gamma, \xi, \eta, \zeta) d \gamma \wedge d \alpha+ \\
+ & \left(r_{15} w+r_{16} w_{1}\right)(\alpha, \beta, z, \xi, \eta, \zeta) d \alpha \wedge d \beta=\iiint_{D_{1}}\left(r_{12} f_{1}+r_{14} f_{2}+r_{16} f_{3}\right)(\alpha, \beta, \gamma, \xi, \eta, \zeta) d \alpha d \beta d \gamma .
\end{aligned}
$$

С учетом (16) получаем

$$
\iint_{G T M Q} u(\xi, \beta, \gamma) d \beta \wedge d \gamma=p_{1}(\xi, \eta, \zeta)
$$


где $p_{1}(\xi, \eta, \zeta)$ выражается через $\mathbf{R}$ и граничные условия (10). Очевидно,

$$
\iint_{G T M Q} u(\xi, \beta, \gamma) d \beta \wedge d \gamma=\int_{y_{0}}^{\eta} \int_{z_{0}}^{\zeta} u(\xi, \beta, \gamma) d \gamma d \beta
$$

Дифференцирование (22) дает

$$
u(\xi, \eta, \zeta)=\frac{\partial^{2} p_{1}(\xi, \eta, \zeta)}{\partial \eta \partial \zeta}
$$

Аналогично, используя третью и пятую строки (14), получаем

$$
v(\xi, \eta, \zeta)=\frac{\partial^{2} p_{2}(\xi, \eta, \zeta)}{\partial \xi \partial \zeta}, \quad w(\xi, \eta, \zeta)=\frac{\partial^{2} p_{3}(\xi, \eta, \zeta)}{\partial \xi \partial \eta} .
$$

При этом в (18) $r_{1 j}$ заменяются на $r_{3 j}$ и $r_{5 j}$ соответственно. То, что формулы $(23)-(24)$ дают решение смешанной задачи, проверяется точно так же, как и в случае задачи Коши.

\section{ЛитерАтУРА}

[1] Бицадзе А.В. О структурных свойствах решений гиперболических систем уравнений с частными производными, Матем. моделирование 6 (6), 22-31 (1994).

[2] Чекмарев Т.В. Формуль решения задачи Гурса для одной линейной системы уравнений с частными производными, Дифференщ. уравнения 18 (9), 1614-1622 (1982).

[3] Плещинская И.Е. Об эквивалентности некоторых классов эллиптических и гиперболических систем первого порядка и уравнений второго порядка с частными производными, Дифференц. уравнения 23 (9), 1634-1637 (1987).

[4] Миронова Л.Б. О методе Римана в $R^{n}$ для одной системы с кратными характеристиками, Изв. вузов. Матем., № 1, 34-39 (2006).

[5] Жегалов В.И., Миронова Л.Б. Об одной системе уравнений с двукратными старшими частными производными, Изв. вузов. Матем., № 3, 12-21 (2007).

[6] Жегалов В.И. Задача с нормалъными производными в граничных условиях для системы дифференциалъных уравнений, Изв. вузов. Матем., № 8, 70-72 (2008).

[7] Воронова Ю.Г. О задаче Коши для линейных гиперболических систем уравнений с нулевыми обобщеннъми инвариантами Лапласа, Уфимский матем. журн. 2 (2), 20-26 (2010).

[8] Жибер А.В., Костригина О.С. Задача Гурса для нелинейных гиперболических систем уравнений с интегралами первого и второго порядка, Уфимский матем. журн. 3 (3), 67-79 (2011).

[9] Созонтова Е.А. О характеристических задачах с нормалъными производными для системъ гиперболического типа, Изв. вузов. Матем., № 10, 43-54 (2013).

[10] Андреев А.А., Яковлева Ю.О. Задача Коши для системь дифференииалънъх уравнений гиперболического типа порядка п с некратными характеристиками, Вестн. Сам. гос. техн. ун-та. Сер. Физ.-матем. науки 21 (4), 752-759 (2017).

[11] Жегалов В.И. Трехмерный аналог задачи Гурса, Неклассические задачи и уравнения смешанного типа, Новосибирск, 94-98 (1990).

[12] Жегалов В.И., Севастьянов В.А. Задача Гурса в четырехмерном пространстве, Дифференц. уравнения 32 (10), 1429-1430 (1996).

[13] Жегалов В.И. О трехмерной функции Римана, Сиб. матем. журн. 38 (5), 1074-1079 (1997).

[14] Миронов А.Н. О построении функции Римана для одного уравнения четвертого порядка, Дифференц. уравнения 37 (12), 1698-1701 (2001).

[15] Уткина Е.А. К общему случаю задачи Гурса, Изв. вузов. Матем., № 8, 57-62 (2005).

[16] Миронов А.Н. Метод Римана для уравнений со старшей частной производной в $R^{n}$, Сиб. матем. журн. 47 (3), 584-594 (2006).

[17] Романовский Р.К. О матрицах Римана первого и второго рода, Матем. сб. 127 (4), $494-501$ (1985).

[18] Романовский Р.К. Экспоненциалъно расщепляемъе гиперболические системъ с двумл независимыми переменными, Матем. сб. 133 (3), 341-355 (1987).

[19] Воробьева Е.В., Романовский Р.К. Метод характеристик для гиперболических краевых задач на плоскости, Сиб. матем. журн. 41 (3), 531-540 (2000). 
[20] Романовский Р.К., Мендзив М.В. Устойчивость решений задачи Коши для гиперболической системъ на плоскости с периодическими по времени коэфбициентами, Сиб. матем. журн. 48 (5), 1134-1141 (2007).

[21] Романовский Р.К., Медведев Ю.А. Оптималъное двустороннее граничное управление теплопереносом в стержне. Гиперболическая моделъ, Изв. вузов. Матем., № 6, 54-60 (2016).

Любовь Борисовна Миронова

Елабужский институт (филиал) Казанского федерального университета, ул. Казанская, д. 89, г. Елабуга, 423604, Россия,

e-mail : lbmironova@yandex.ru

\section{L.B. Mironova}

\section{Application of Riemann method to one system in three-dimensional space}

Abstract. For a system of three equations of the second order we prove existence and uniqueness of solutions to the Cauchy problem and to problem with conditions on characteristics and a free surface. We construct solutions to these problems in terms of the Riemann matrix.

Keywords: hyperbolic system, Riemann method, Cauchy problem, characteristics.

Lyubov' Borisovna Mironova

Elabuga Institute of Kazan Federal University,

89 Kazanskaya str., Elabuga, 423600 Russia,

e-mail : lbmironova@yandex.ru 